\title{
An unusual presentation of posterior reversible encephalopathy syndrome-a case report
}

\author{
K. Soundarya Rajeshwari ${ }^{*}$, Vishaka Agarwal, S. Satish and K. T. Jayakumar
}

\begin{abstract}
Background: Acute neurological emergencies in pregnant and postpartum women presenting as headache, visual disturbances, seizures, and elevated blood pressure are usually attributed to preeclampsia and eclampsia. However cerebral venous sinus thrombosis (CVST) and posterior reversible encephalopathy syndrome (PRES) are two important, rare conditions which should be considered. PRES is a rare neuroradiological syndrome characterised by vasogenic oedema of subcortical white matter, commonly involving posterior cerebral hemispheres. Preeclampsia and eclampsia are the common causes of PRES. However, late postpartum eclampsia concurrent to PRES is rare. Here, we report a rare case of PRES secondary to late postpartum eclampsia with atypical radiological findings.
\end{abstract}

Case presentation: A 25-year-old married woman with prior history of two spontaneous abortions presented to us on day 5 postpartum, post lower segment caesarean section (LSCS) with complaints of headache followed by one episode of new onset generalised tonic-clonic seizures. There was no history of seizures prior to the pregnancy and no history of hypertension or diabetes during any of her pregnancies. On examination, BP was 140/90 mm Hg and PR 98 bpm. Systemic examination revealed GCS 15/15, no focal neurological deficits, and bilateral flexor plantar response. Fundus examination was normal with no evidence of hypertensive retinopathy. Preliminary haematological and biochemical tests including antiphospholipid antibody (APLA) profile were done in view of prior abortions and were within normal limits. Lumbar puncture was done-normal study. EEG was normal. MRI with MRV was suggestive of atypical posterior encephalopathy. In view of late onset postpartum seizure, the patient was started on injection of magnesium sulphate, anti-hypertensives, and antiepileptics; following which, she improved and was discharged.

Conclusion: Though preeclampsia and eclampsia are usually screened entities, we should also follow women in puerperium for late postpartum eclampsia which is defined as seizures beyond $48 \mathrm{~h}$ of delivery up to 4 weeks postpartum. So it is important to follow-up postpartum women for complications like PRES because early detection and treatment can lessen the morbidity and mortality as they are completely reversible. Thus, health care professionals should be educated about the same. Apart from antenatal and intrapartum care, postpartum care should also be given equal importance.

Keywords: Posterior reversible encephalopathy syndrome (PRES), Late postpartum eclampsia, Seizures, Magnetic resonance imaging, Magnesium sulphate, Case report

\footnotetext{
* Correspondence: soundarya.reddy15@yahoo.com

SRM Institute of Science and Technology, Potheri, Kattankulathur,

Chengalpattu District, Tamilnadu 603203, India
}

\section{Springer Open}

(c) The Author(s). 2021 Open Access This article is licensed under a Creative Commons Attribution 4.0 International License, which permits use, sharing, adaptation, distribution and reproduction in any medium or format, as long as you give appropriate credit to the original author(s) and the source, provide a link to the Creative Commons licence, and indicate if changes were made. The images or other third party material in this article are included in the article's Creative Commons licence, unless indicated otherwise in a credit line to the material. If material is not included in the article's Creative Commons licence and your intended use is not permitted by statutory regulation or exceeds the permitted use, you will need to obtain permission directly from the copyright holder. To view a copy of this licence, visit http://creativecommons.org/licenses/by/4.0/. 


\section{Background}

Posterior reversible encephalopathy syndrome (PRES) is an acute neuroradiological entity presenting with nonspecific signs and symptoms such as headache, altered mental status, seizures, visual disturbances in the form of cortical blindness, and focal neurological deficits [1, 2]. Typical radiological findings show oedema of the posterior cerebral regions that is the parieto-occipital lobes. However, atypical PRES presents with involvement of anterior cerebral regions and deep white matter [3]. Preeclampsia, eclampsia, infection, sepsis, shock, autoimmune disease, cancer chemotherapy, hypertension, and solid organ or bone marrow transplantation are the predominant causes described in association with PRES [4]. Preeclampsia and eclampsia are the most common causes of PRES.

Hereby, reporting a rare case of late postpartum eclampsia with atypical radioimaging, diagnosed to be PRES.

\section{Case presentation}

A 25-year-old postpartum female presented to us with history of generalised tonic-clonic seizures lasting for 2 min duration associated with tongue bite and loss of consciousness preceded by headache lasting for half an hour.

Patient was apparently alright 4 days ago when she developed first episode of GTCS on day 5 postpartum following which she was hospitalised outside and evaluated for the same with MRI and MRV which were normal. Vitals, routine blood and urine examination, CSF analysis, and EEG were normal. Patient was started on antiepileptics (levetiracetam $500 \mathrm{mg}$; fosphenytoin $150 \mathrm{mg}$ intravenously twice a day), despite which she continued to have seizures with preceding headache; in view of which, she was referred to our hospital for further workup. There was no history of weakness, visual disturbance, fever, neck stiffness, and trauma.

Her antenatal period was uneventful. There was no history of diabetes or hypertension during pregnancy, no previous episodes of seizures, and no history of pedal oedema during pregnancy. She was diagnosed to have hypothyroidism during pregnancy and was on tablet thyroxine $12.5 \mu \mathrm{g}$ supplementation. She had history of two first trimester abortions (missed abortion followed by a spontaneous abortion). However, she was not evaluated for antiphospholipid antibody syndrome (APLAS). Her present pregnancy was full term, and she had undergone LSCS in view of cephalopelvic disproportion.

On general examination, patient was conscious, oriented, afebrile, well built, and nourished. LSCS scar was healthy. Vitals on admission showed a blood pressure of $140 / 90 \mathrm{~mm} \mathrm{Hg}$, heart rate of 82 per minute, saturating at $100 \%$ on room air, and capillary blood glucose of 102 $\mathrm{mg} / \mathrm{dl}$. Systemic examination including the central nervous system examination was unremarkable. Antiepileptics were continued. Baseline investigations were repeated including MRI with MRV after neurologist consultation. MRI with MRV showed multiple areas of T2/FLAIR hyperintensities in subcortical and white matter areas of bilateral occipital, posterior parietal, and right frontal regions; superficial cortical sinuses appeared normal, features suggesting of atypical posterior encephalopathy. During the course of hospital stay, patient developed another episode of focal seizures and complained of left upper and lower limb numbness. Her blood pressure recordings were noted to be high (ranging from $130 / 70$ to $180 / 120 \mathrm{~mm} \mathrm{Hg}$ ).

Patient was diagnosed to have late postpartum eclampsia with suspected PRES and was started on injection of magnesium sulphate, injection labetolol, and tablet nifedipine along with antiepileptics (levetiracetam $500 \mathrm{mg}$; fosphenytoin $150 \mathrm{mg}$ intravenously twice a day). Gynaecologist consultation was sought. Ophthalmologist consultation was obtained, and there was no evidence of hypertensive retinopathy/papilledema. Cardiologist consultation was obtained, and screening echo was normal. Nephrologist's opinion was obtained, and renal artery Doppler was suggested and it was normal. Rheumatologist's consultation was obtained for prior first trimester abortions to rule out connective tissue disorder and was advised ANA, Anti-dsDNA, beta-2 glycoprotein, lupus anticoagulant, anticardiolipin antibody which were normal. Following magnesium sulphate injections and antihypertensives, patient's blood pressure was under control, and there were no further episodes of seizures; hence, patient was discharged after 10 days of admission, with advice of continuing antihypertensives and antiepileptics (levetiracetam $500 \mathrm{mg}$ twice a day).

Patient returned to us after 1 week; she was asymptomatic, and blood pressure was 100/60 mm Hg. Repeat MRI was done which showed resolution of previous signs hence diagnosis of PRES was confirmed.

\section{Investigations}

\section{Admission MRI images}

Admission MRI images are shown in Figs. 1, 2, 3, 4, 5 and 6 .

\section{Follow-up images after 15 days}

Figures 7, 8, and 9 show the follow-up images after 15 days.

\section{Discussion}

Preeclampsia and eclampsia are hypertensive disorders of pregnancy complicating around 10\% of all pregnancies with significant maternal and foetal morbidity and mortality [5]. Preeclampsia is characterised by 


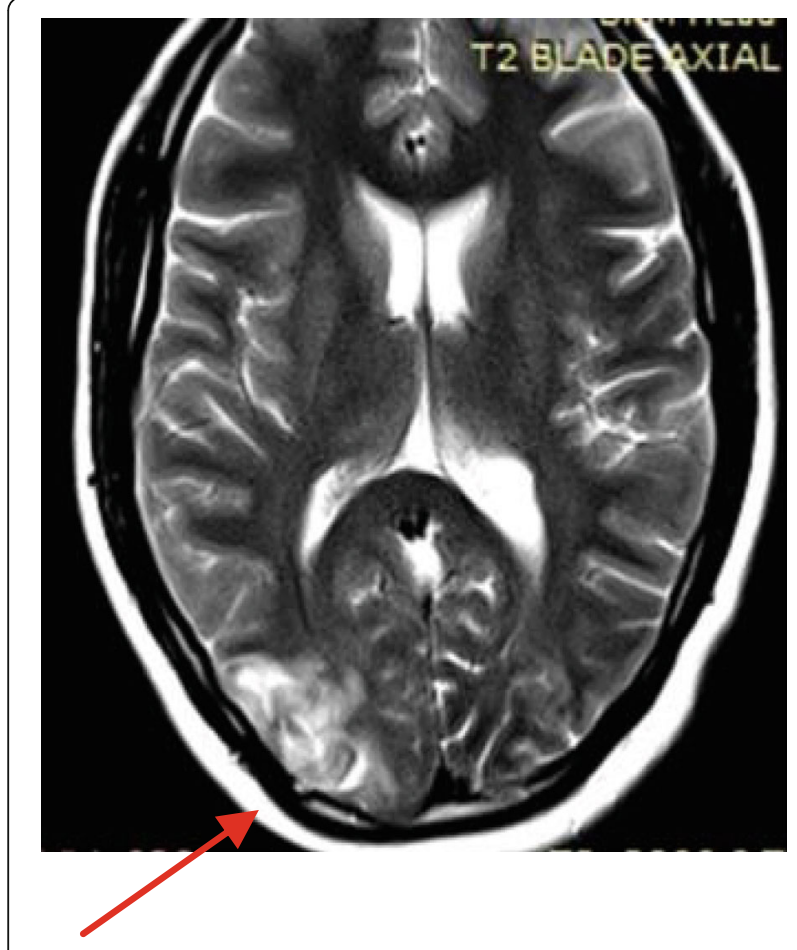

Fig. 1 T2 ill-defined hyperintensities in the right occipital lobe

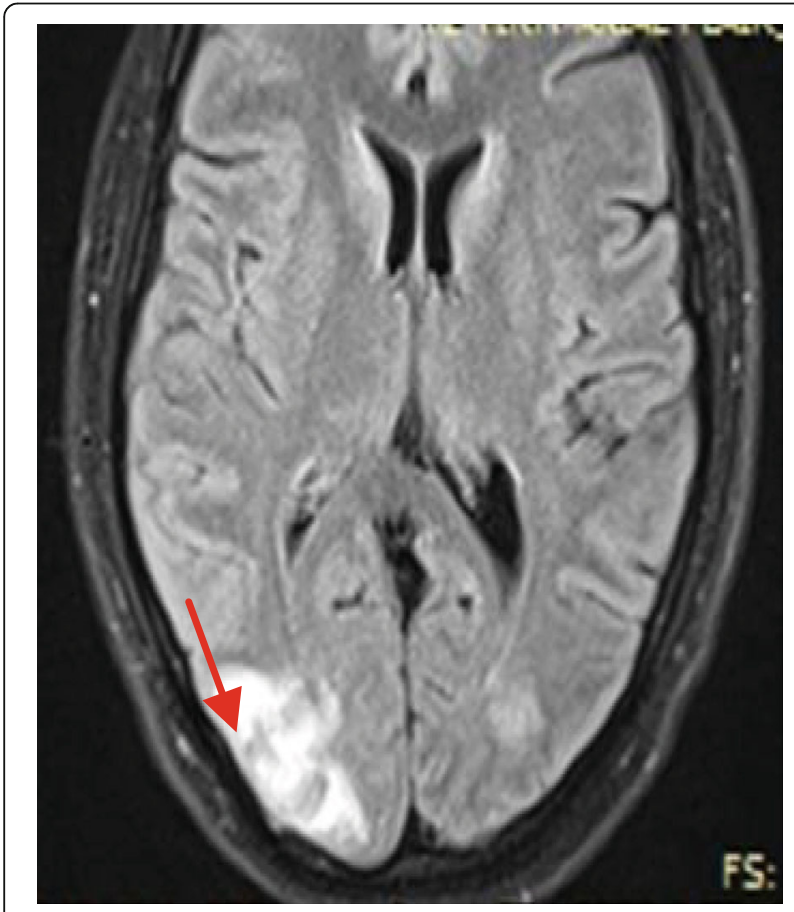

Fig. 2 FLAIR ill-defined hyperintensities in the right occipital lobe

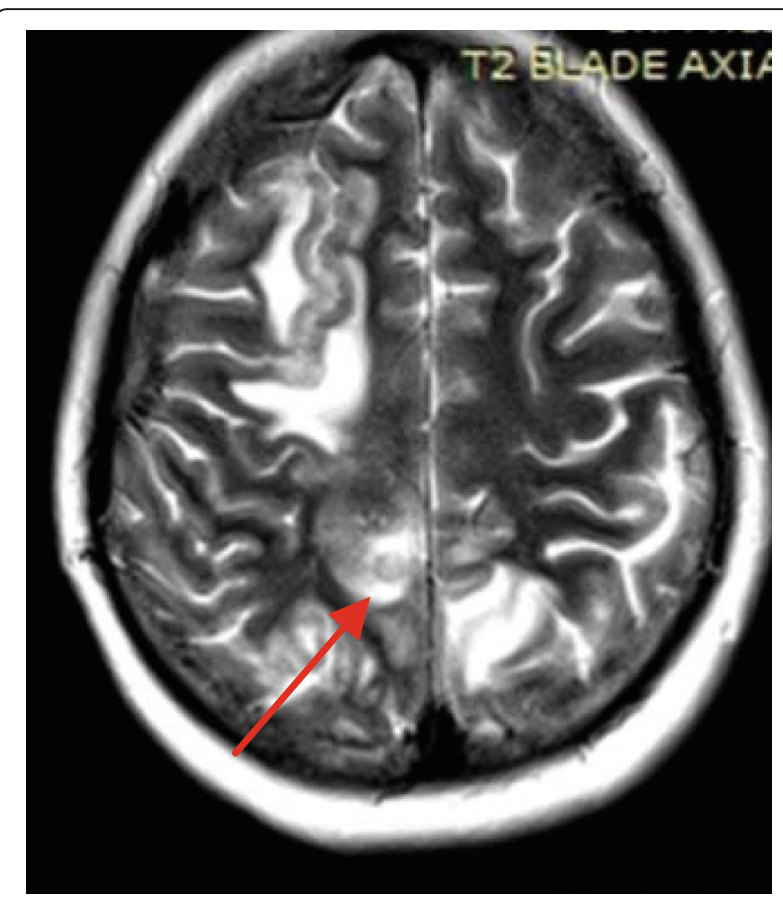

Fig. 3 T2 ill-defined hyperintensities in parietofrontal lobes right more than left

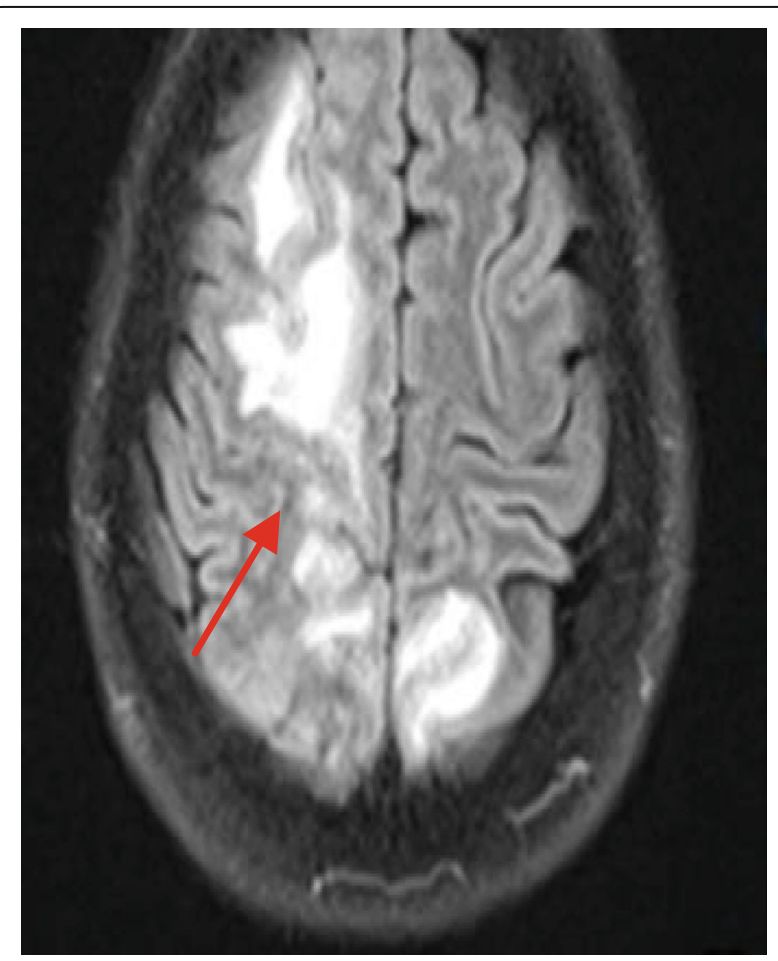

Fig. 4 FLAIR ill-defined hyperintensities in parietofrontal lobes right more than left 


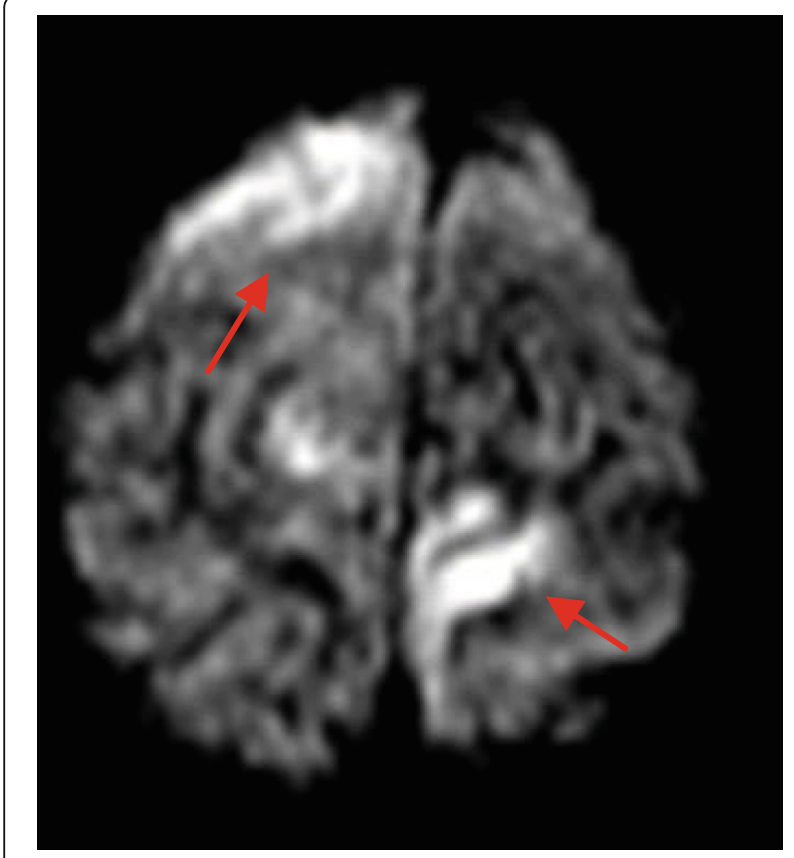

Fig. 5 Diffusion restriction in bilateral frontal and parietal lobes respectively

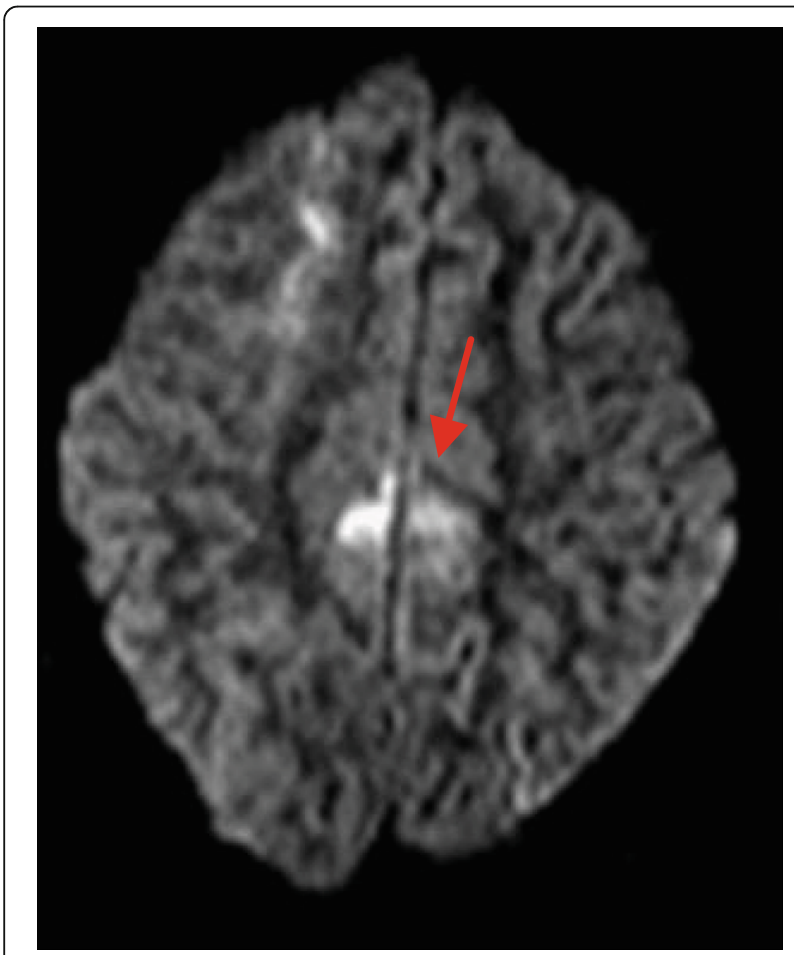

Fig. 6 Diffusion restriction in bilateral frontal and parietal lobes respectively

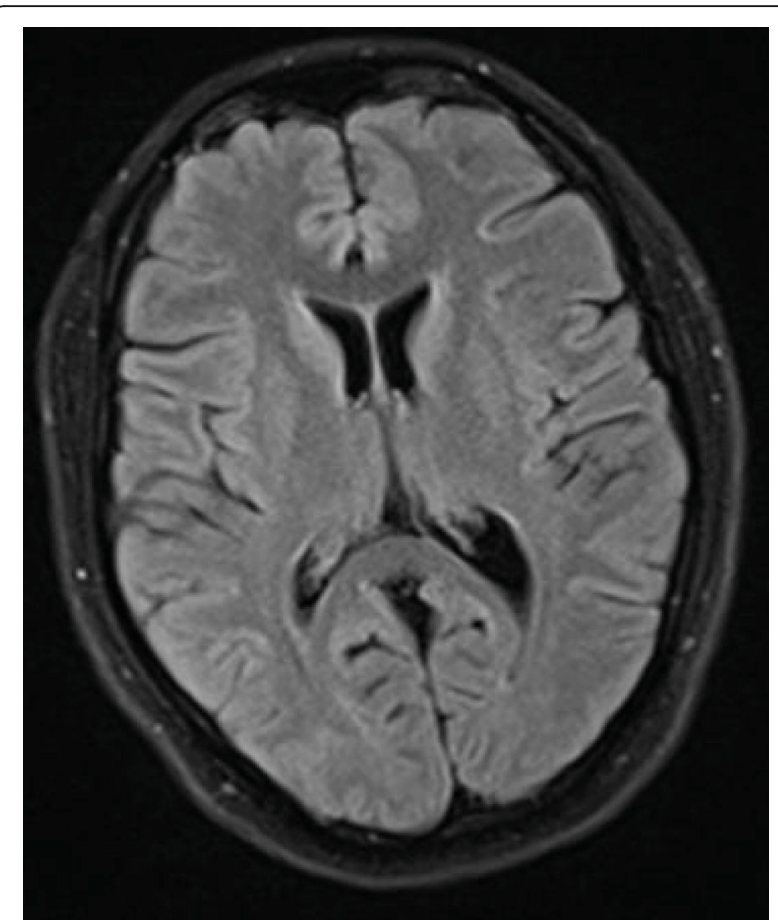

Fig. 7 T2 FLAIR MRI image showing complete resolution of frontal and occipital hyperintensities

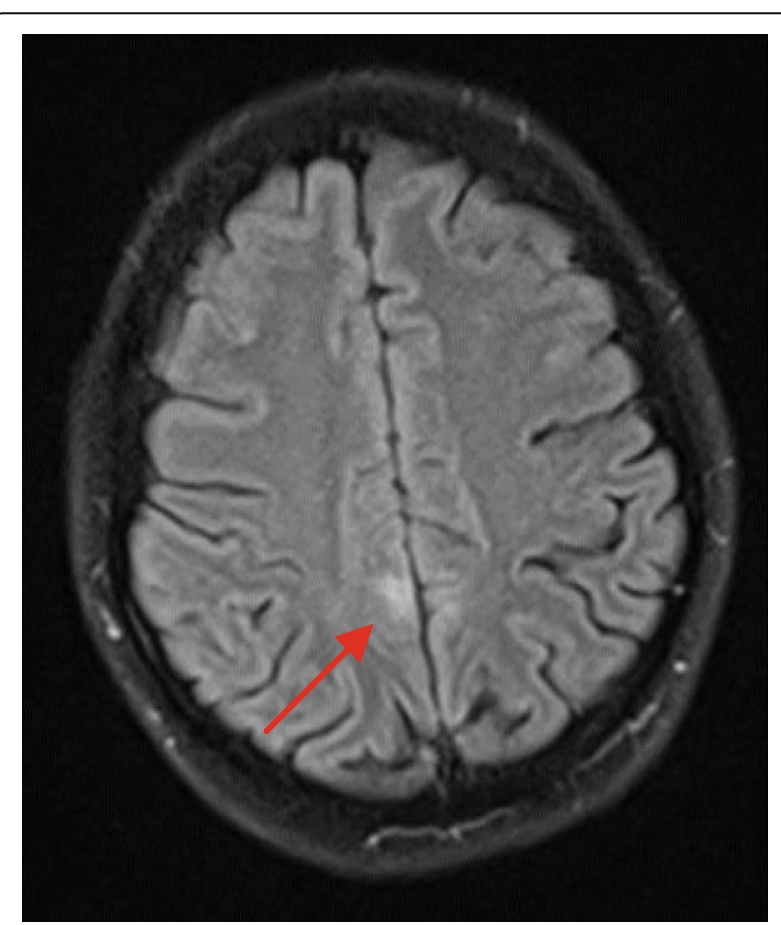

Fig. 8 Resolving T2 FLAIR and DWI images showing diffusion restriction in parietal lobe 


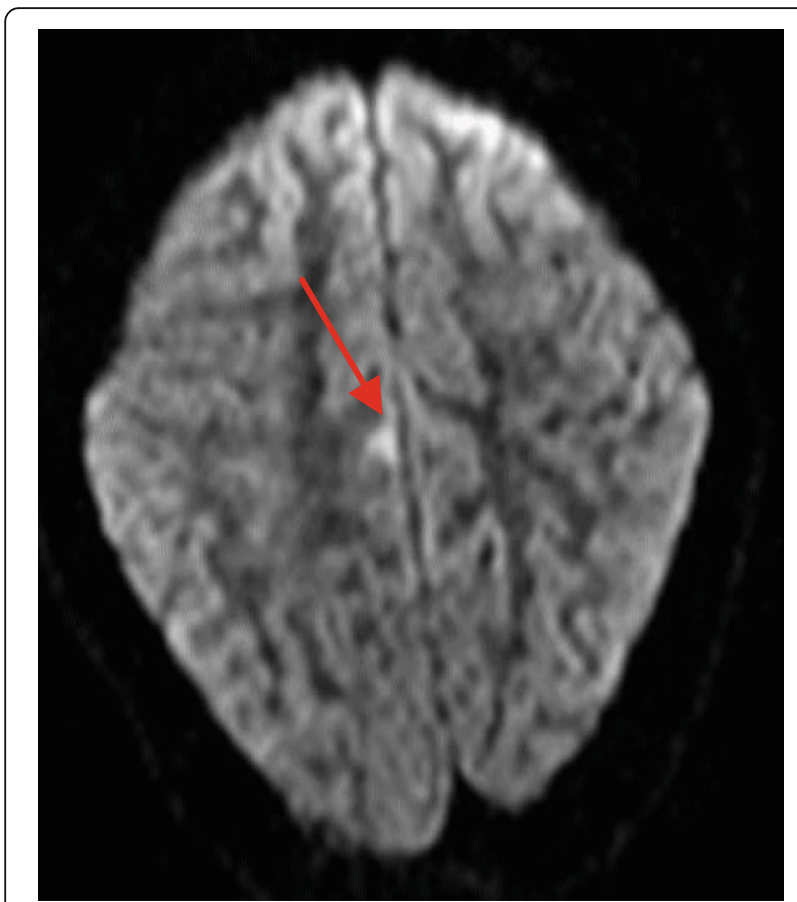

Fig. 9 Resolving T2 FLAIR and DWI images showing diffusion restriction in parietal lobe

hypertension after 20 weeks of gestation in a previously normotensive individual with signs and symptoms of target organ injury. Eclampsia is abrupt development of seizures or coma during the gestational period or postpartum not attributable to any other cause [6]. Usually, it occurs between 20 weeks of pregnancy and $48 \mathrm{~h}$ postpartum. A few cases occur from $48 \mathrm{~h}$ to 4 weeks postpartum called as late postpartum eclampsia or preeclampsia [7]. PRES with late onset eclampsia is a rarely encountered entity; as a result, most of the clinicians are unaware about the same [8].

In more than $90 \%$ of delayed presentation of postpartum eclampsia, there will at least be one early missed symptom suggesting preeclampsia which usually goes unreported by the patient to the treating physician; this prevents the diagnosis and treatment of the same, leading to increased risk of onset of PRES $[9,10]$.

\section{Conclusion}

As stated above, PRES can manifest as late postpartum eclampsia without prior evidence of preeclampsia and eclampsia during antenatal period. It is of utmost importance that the treating physician is aware of such condition, so that prompt diagnosis and early treatment is administered to reduce maternal morbidity and mortality as PRES is reversible.

\section{Limitations}

Angiographic study was not done to rule out vascular pathology.

\begin{abstract}
Abbreviations
CVST: Cerebral venous sinus thrombosis; PRES: Posterior reversible encephalopathy syndrome; LSCS: Lower segment caesarean section; BP: Blood pressure; PR: Pulse rate; GCS: Glasgow coma score; APLA: Antiphospholipid antibody; EEG: Electroencephalogram; GTCS: Generalised tonic-clonic seizures; CSF: Cerebrospinal fluid; MRI: Magnetic resonance imaging; MRV: Magnetic resonance venography; T2-FLAIR: T2-weighted-fluid-attenuated inversion recovery; ANA: Antinuclear antibody; dsDNA: Double-stranded deoxyribonucleic acid; DWI: Diffusionweighted imaging
\end{abstract}

\section{Acknowledgements}

Not applicable.

\section{Authors' contributions}

KSR, KTJ, and SS diagnosed the patient and collected clinical data. KSR, VA, SS, and KTJ carried out the literature search, review, and manuscript preparation. All authors read and approved the final manuscript.

\section{Authors' information}

KSR-MD, internal medicine resident

VA-MD, internal medicine resident

SS-DM, neurology resident

KTJ-MD, professor of internal medicine

\section{Funding}

Not applicable.

\section{Availability of data and materials}

The data sets supporting the conclusion of this article are included within the article.

\section{Ethics approval and consent to participate}

We confirm that ethical clearance was not required for publication of this case report.

\section{Consent for publication}

Written informed consent to publish this information was obtained from study participant.

\section{Competing interests}

Authors confirm that they have no competing interests.

Received: 21 August 2020 Accepted: 23 November 2020

Published online: 07 January 2021

\section{References}

1. Fischer M, Schmutzhard E. Posterior reversible encephalopathy syndrome. J Neurol. 2017;264(8):1608-16. https://doi.org/10.1007/s00415-016-8377-8.

2. Striano $P$, Striano $S$, Tortora F, et al. Clinical spectrum and critical care management of posterior reversible encephalopathy syndrome (PRES). Med Sci Monit. 2005;11(11):CR549-53.

3. Staykov D, Schwab S. Posterior reversible encephalopathy syndrome. J Intensive Care Med. 2012;27(1):11-24. https://doi.org/10.1177/ 0885066610393634.

4. Sudulagunta SR, Sodalagunta MB, Kumbhat M, Settikere Nataraju A. Posterior reversible encephalopathy syndrome (PRES). Oxf Med Case Rep. 2017;2017(4):om×011. https://doi.org/10.1093/omcr/omx011 Published 2017 Apr 3.

5. Hutcheon JA, Lisonkova S, Joseph KS. Epidemiology of pre-eclampsia and the other hypertensive disorders of pregnancy. Best Pract Res Clin Obstet Gynaecol. 2011;25(4):391-403. https://doi.org/10.1016/j.bpobgyn.2011.01.006

6. Peres GM, Mariana M, Cairrão E. Pre-eclampsia and eclampsia: an update on the pharmacological treatment applied in Portugal. J Cardiovasc Dev Dis. 2018;5(1):3. https://doi.org/10.3390/jcdd5010003 Published 2018 Jan 17.

7. Chhabra S, Tyagi S, Bhavani M, Gosawi M. Late postpartum eclampsia. J Obstet Gynaecol. 2012;32(3):264-6. https://doi.org/10.3109/01443615.2011.639467. 
8. Zhang L, Wang Y, Shi L, Cao J, Li Z, Wáng YX. Late postpartum eclampsia complicated with posterior reversible encephalopathy syndrome: a case report and a literature review. Quant Imaging Med Surg. 2015;5(6):909-16. https://doi.org/10.3978/j.issn.2223-4292.2015.12.04.

9. Chames MC, Livingston JC, Ivester TS, Barton JR, Sibai BM. Late postpartum eclampsia: a preventable disease? Am J Obstet Gynecol. 2002;186(6):1174-7. https://doi.org/10.1067/mob.2002.123824.

10. Martin J, Sidman R. Late postpartum eclampsia: a common presentation of an uncommon diagnosis. J Emerg Med. 2003;25(4):387-90. https://doi.org/ 10.1016/s0736-4679(03)00240-3

\section{Publisher's Note}

Springer Nature remains neutral with regard to jurisdictional claims in published maps and institutional affiliations.

\section{Submit your manuscript to a SpringerOpen ${ }^{\circ}$ journal and benefit from:}

- Convenient online submission

- Rigorous peer review

- Open access: articles freely available online

High visibility within the field

- Retaining the copyright to your article

Submit your next manuscript at $\boldsymbol{\nabla}$ springeropen.com 